\title{
BEAM PROFILE WIRE-SCANNER/HALO-SCRAPER SENSOR ANALOG INTERFACE ELECTRONICS*
}

\author{
M. E. Gruchalla, J. O’Hara, Honeywell FM\&T/NM, Albuquerque, NM, 87185, USA \\ D. Barr, T. Cote, L. Day, D. Gilpatrick, M. Stettler, Los Alamos National Laboratories, \\ Los Alamos, NM, 87545, USA \\ D. Martinez, General Atomics, Los Alamos, 87545, USA
}

\begin{abstract}
The halo experiment presently being conducted at the Low Energy Demonstration Accelerator (LEDA) at Los Alamos National Laboratory utilizes a generally traditional wire scanner for measurement of the beam core profile and a graphite scraper for measurement of the tails of the beam distribution. A lossy integrator is used to detect the replacement charge flowing to the wire and scraper. Independent programmable dc-bias voltages are applied to the wire and the scraper through the analog electronic interface to optimize charge capture from the two sensors. A programmable guard voltage is applied to isolate the scraper from the resistivity of the cooling system. Programmable gain provides a total dynamic range in the analog electronics of greater than about one part in $10^{6}$. The analog signal is digitized to 14 bits plus sign, and the equivalent input noise is nominally $30 \mathrm{fC}$.
\end{abstract}

\section{WIRE-SCANNER/HALO-SCRAPER SIGNALS}

The signal from the wire-scanner/halo-scraper (WS/HS) sensors is the charge required to be delivered to the sensor to replace the charge imbalance caused by the interaction of the sensor with the accelerator beam [1]. Secondary electrons are radiated from the wire and protons are collected in the scraper in the proton beam of the LEDA. Therefore, the charge that must be delivered to both sensors to maintain charge balance in normal operation is an electron current to the sensor.

It is reasonable to expect that under various conditions of operation, for example different $\mathrm{Z}$ positions and various accelerator tunes, the WS/HS sensors will collect other particles. Although it is not intended that these particles be collected, it is important to know that they are collected. The charge of these particles may be either positive or negative. Therefore, the displacement current may be an electron current either to or from the sensor. To provide maximum versatility in the analog-front-end electronics (AFE) collecting the sensor signals, the AFE must be capable of processing bipolar input signals.

The displacement charge collected is a function of both the intensity of the illumination and the duration of the illumination. The secondary-emission current from the wire sensor is a small percentage of the beam current. Microampere wire currents result from milliampere beam currents. The nominal macropulse width in the LEDA is
$30 \mu \mathrm{s}$. The collected wire charge for the beam currents of nominally $100 \mathrm{~mA}$ is on the order of $10 \mathrm{mC}$ at the beam core. The maximum scraper signal is substantially higher than the wire signal, but the scraper is intended to collect charge information in the outer tails of the distribution to the limits of the beam pipe. Therefore, the scraper actually presents with both the highest and lowest signals. Therefore, the scraper sensor has the more demanding dynamic-range requirements.

\section{ELECTRONIC INTERFACE}

A block diagram of two AFE channels is shown in Figure 1. The input stage is configured as a lossy integrator. The integrating capacitance provides the integration constant, and the shunt resistance provides integrator reset. The response pole is set at nominally $160 \mathrm{~Hz}(1 \mathrm{~ms}$ reset time constant) which can reasonably accommodate an accelerator pulse-repetition frequency as high as $20 \mathrm{~Hz}$. The AFE is provided with three integration constants roughly at decade intervals and remotely selectable under program control. The response poles for all three integration constants are made equal so that the frequency response is consistent among the three integration values.

The macropulses comprise a substantial RF component from the beam micropulses. The RF component is of no particular interest in the WS/HS measurements, but it must be accommodated to assure that the desired signals are not compromised. The RF component is terminated at the AFE input by means of a passive surge termination with a comparatively high response pole. The response pole of this surge termination is accommodated in the lossy integrator by adding a corresponding lead (response zero) in the integrator function. The combined result of the surge termination and the lossy integrator is a continuous integration function over the length of the macropulse. The lossy integrator is followed by a programmable-gain stage which is also under program control. The combination of the integration and gain control provides a total full-scale charge range of about 500:1.

The gain stage is followed by an absolute-value stage. This allows a unipolar 14-bit analog-to-digital converter to be utilized. A sign bit is provided output from the absolute value stage so that the total digitized resolution is 14 bits plus sign.

\footnotetext{
* Supported by US DOE, Office of Defense Programs, and the

Office of Nuclear Energy, Science and Technology.
} 


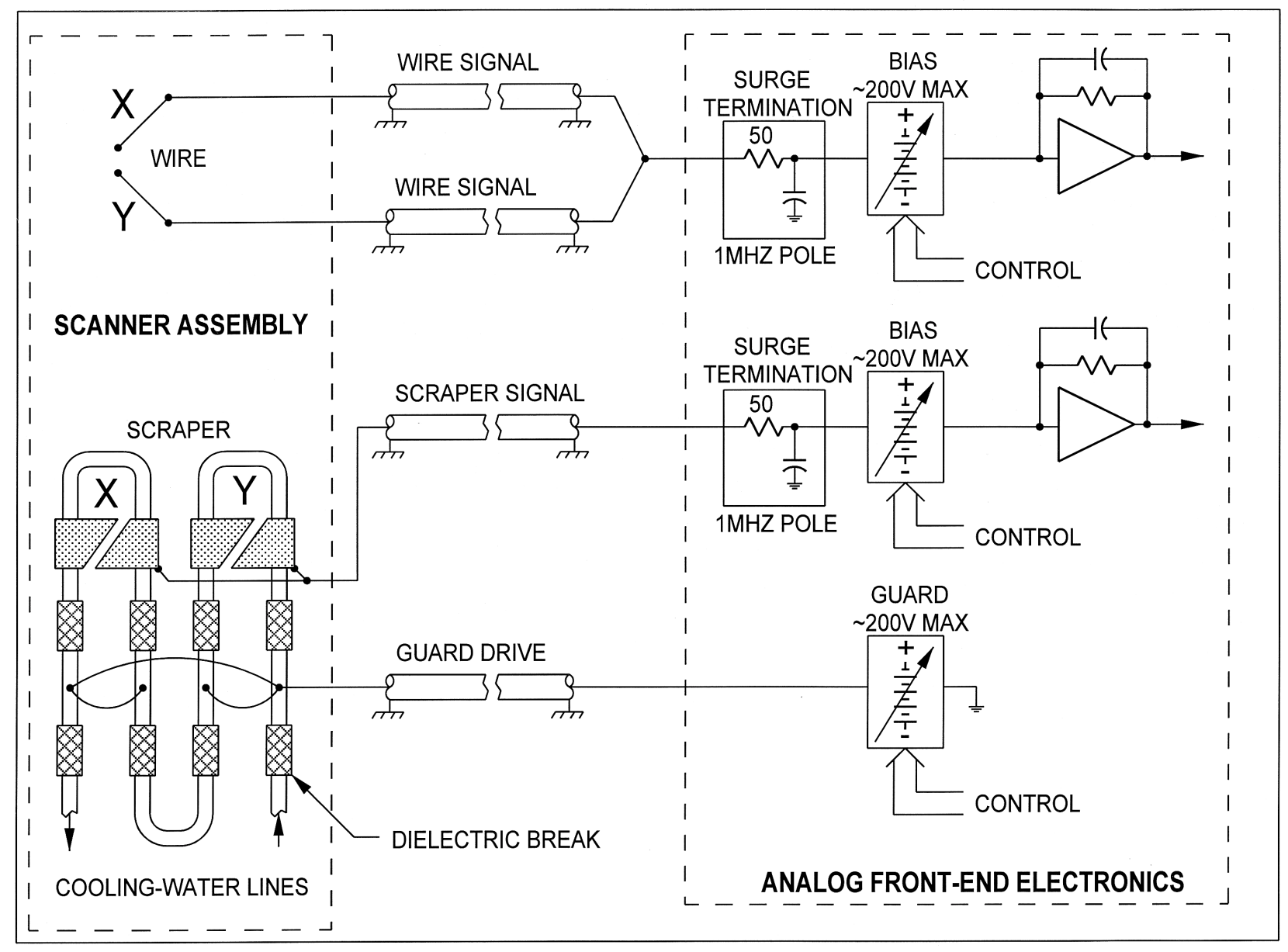

Figure 1. LEDA Wire-Scanner/Halo-Scraper AFE Block Diagram

\section{CHARGE-EQUIVALENT NOISE}

\subsection{Fundamental Amplifier Noise}

The fundamental minimum measurement limit is set by the input charge-equivalent noise of the AFE. This noise is predominately first-principle noise [1]. The tangentialnoise is the most appropriate noise specification since instantaneous measurements are recorded [2,3]. The computed tangential charge-equivalent input noise of the AFE configured for maximum gain is nominally $10 \mathrm{fC}$. In the final application configuration, the measured noise is nominally 30fC. The overall full-scale signal for the actual fielded AFE configuration is $1.5 \mu \mathrm{C}$. The overall measurement dynamic range of the AFE is greater than six orders of magnitude in the fielded configuration. Also, the measurement strategy includes making two measurements of the integrated signal separated by several microseconds, and the relative current computed from the difference in integrated charge at the two points. This difference measurement reduces effective noise and eliminates errors due to dc offsets.

\subsection{Excess-Noise}

In addition to the fundamental AFE noise, various noise sources in the accelerator infrastructure can contaminate the measurements. For example, one severe noise source discovered in the LEDA was the WS/HS stepper-motor drive current. This is a pulse-width-modulated current which is present even when the motor is stationary. This signal resulted in a very large corrupting noise signal. This particular noise was eliminated by removing the motor drive current from all WS/HS motors during data acquisition.

Other potential sources of excess noise include AC Mains common-mode potentials between the accelerator structure and the data-acquisition electronics, conducted and radiated noise from support equipments such as vacuum pumps, water pumps, warning lights, overhead lighting (particularly electronic florescent-lamp ballasts) as well as RF leakage from the accelerator.

The overall equivalent input noise achieved in the WS/HS measurements at the LEDA is nominally $200 \mathrm{fC}$ tangential and the full-scale signal $1.5 \mu \mathrm{C}$. 


\section{SENSOR BIAS}

\subsection{Bias Voltage}

A dc bias field must be provided to optimize sensor performance. This bias is provided directly to the sensors by the AFE. The bias is applied directly in series with the low-level signal. Therefore, the bias source must exhibit extremely low noise. Further, the common-mode coupling to noise sources such as the digital elements of the AFE must be extremely small. Since the bias current required to bias the sensors is very small, a programmable battery unit was chosen as the bias means. Each AFE signal channel comprises a programmable bias battery in series with the signal path. The battery polarity is programmable, and the battery voltage is programmable from $0 \mathrm{~V}$ to nominally $200 \mathrm{~V}$.

\subsection{Scraper Guarding}

The wire sensor is a totally floating sensor. When a bias voltage is applied, effectively no bias current flows in the signal path, and no substantial excess noise is introduced. The scraper sensor is water cooled. Although the scraper is isolated from the water system with dielectric isolators, the water channel is a resistive leakage path (about $50 \mathrm{M} \Omega$ to ground for the polished deionized water in the cooling system) which would result in a substantial bias current when a bias is applied to the scraper. Also, when bias is applied, the turbulent flow in the isolators results in modulation of the water resistance in turn resulting in modulation of the scraper bias current which presents as a substantial excess-noise signal.

The excess noise that would result from conventional biasing of the scraper is substantially reduced by the use of a guarding system. The guard comprises a second set of dielectric isolators to allow the application of a guard voltage to the scraper-sensor water circuit (see Figure 1). When a bias voltage is applied to the scraper, an equal guard voltage is applied to the guard electrodes. This results in a zero voltage potential across the dielectric isolators of the scraper. With this guard configuration, the scraper bias current is reduced to zero. Since there is no potential across the scraper dielectric isolators, there is no modulation noise introduced by the turbulent water flow.

The bias and guard batteries for each data channel are integrated into a single shielded battery assembly which mounts under and directly to the AFE printed-circuit board. Although integrated in a common assembly, the bias and guard batteries are totally independent with independent control.

\section{AFE MECHANICAL ASSEMBLY}

The complete AFE module is shown in Figure 2. The AFE module is implemented in a single-wide VXI module. Each AFE module comprises four data channels with each data channel providing both bias and guard voltages. All data channels are identical and programmable. Each data channel may be used as either a wire channel or a scraper channel. Five AFE modules (20 channels) are utilized in the LEDA experiment.

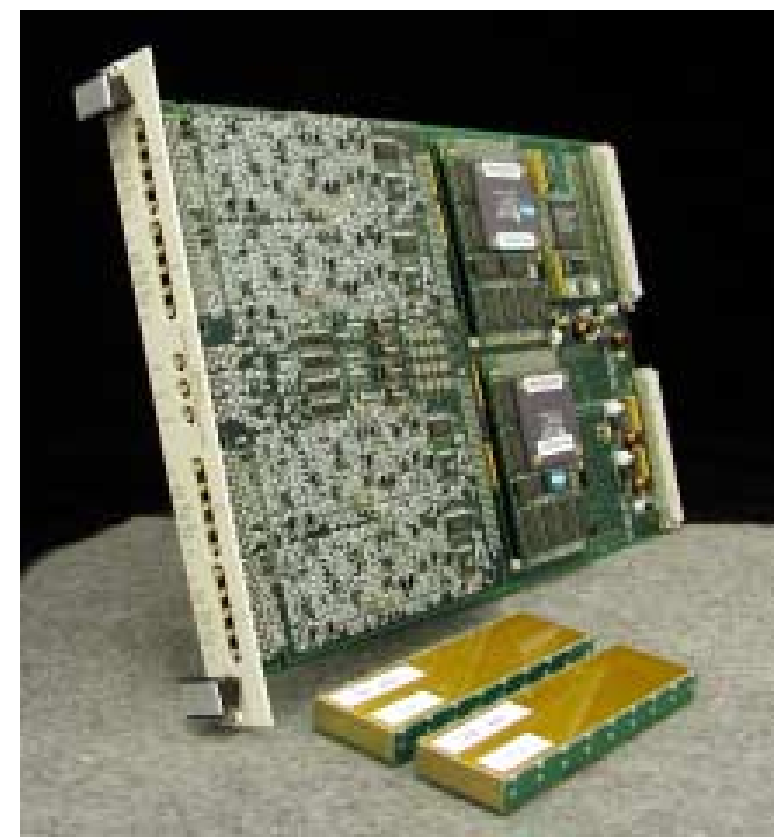

Figure 2. Four-Channel AFE Module With Two of the Four Battery Units Shown

\section{SUMMARY}

The AFE lossy integrator stage integrates the input charge from the WS/HS sensors and provides automatic reset. A linear gain stage provides additional gain for recording low-level signals. The basic equivalent input noise achieved is nominally $\sim 30 \mathrm{fC}$, and the total noise in the fielded configuration is about 200fC. The full-scale gain is programmable over a range of greater than 500:1. The total dynamic range is greater than six orders of magnitude. Independent programmable bias voltages are applied to both wire and scraper sensors. A programmable guard voltage is applied to the scraper to minimize noise contributions from the scraper cooling system. The magnitude of the analog signal is digitized to 14 bits. The sign of the signal is also reported as an output signal. This provides full 14 bits plus sign digital output data for each AFE channel.

\section{REFERENCES}

[1]. D. Gilpatrick, D. Barr, L. A. Day, D. M. Kerstiens, M. Stettler, R. Valdiviez, M. Gruchalla, J. F. O'Hara, J. H. Kamperschroer, "Beam-Profile Instrumentation for BeamHalo Measurement: Overall Description and Operation," PAC 2001, Chicago, June, 2001.

[2] C. D. Motchenbacher and F. C. Fitchen, Low-Noise Electronic Design, John Wiley, New York, 1973.

[3] M. E. Gruchalla, "Measure Wide-Band Noise Using a Standard Oscilloscope," EDN, June, 1980, pp 157-160.

[4] H. E. Green, "A Theoretical Examination of Signal to Noise Ratio," IEEE Transactions on Microwave Theory and Techniques, Vol. 39, No. 3, March 1991, pp 566-567. 